\title{
A comparative study on the effects of oral amiodarone and trimeprazine, two in vitro retinyl ester hydrolase inhibitors, on the metabolic availability of vitamin $A$ in rats
}

\author{
Rainer Schindler ${ }^{1} *$, Tanja Fielenbach ${ }^{1}$ and Gerhard Rave ${ }^{2}$ \\ ${ }^{1}$ Department of Human Nutrition and Food Science, Christian-Albrechts-University zu Kiel, D-24 116, Germany \\ ${ }^{2}$ Institute for Variationsstatistik, Christian-Albrechts-University zu Kiel, D-24 116, Germany
}

(Received 18 November 2004 - Revised 21 March 2005 - Accepted 1 April 2005)

\begin{abstract}
Amiodarone, an antiarrhythmic drug, and trimeprazine, an antipsychotic drug, are both in vitro inhibitors of retinyl ester hydrolase. To determine whether these agents have deleterious effects on aspects of vitamin A metabolism, Brown Norway rats $(n 18)$ were treated at clinically equivalent doses once daily for $26 \mathrm{~d}$ with either oral drug. On day 27, a tolerance test was used to determine whether these agents interfered with vitamin absorption. During the first $8 \mathrm{~d}$, the plasma retinol level declined in all animals. Between days 12 and 27, it rose to near pre-treatment concentrations in the control and trimeprazine groups and remained relatively constant at low levels $(P<0.001)$ in the amiodarone group. The intestinal absorption of vitamin A was reduced $(P<0.05)$ in the amiodarone group compared with the placebo and trimeprazine groups, which did not differ significantly from each other. At the end of the 4-week treatment period, hepatic retinyl ester hydrolase activity was lower in the drug-dosed rats ( $P=0.06$ for amiodarone) than in the controls. With regard to effects on liver reserves, drug treatment resulted in vitamin A depletion $(P<0.019)$, and distinctive patterns of retinol and its esters were seen in response to dosing. In conclusion, amiodarone and trimeprazine have been shown to influence different aspects of retinoid metabolism, namely absorption, storage and transport. In clinical practice, the routine unmonitored use of these drugs and the suggestion that these agents be taken with meals are not recommended.
\end{abstract}

Amiodarone-vitamin A interaction: Trimeprazine-vitamin A interaction: Retinyl ester hydrolase

Vitamin A is a fat-soluble vitamin present in animal-based diets as retinyl esters (retinol esterified to fatty acids; Schindler et al. 1987). Once absorbed, liberated retinol is converted by the activity of acyltransferase back to esterified retinol, in which form vitamin A is targeted to the liver, its primary site of storage (Helgerud et al. 1982; MacDonald \& Ong, 1988). Central to vitamin A handling by the organism is the reversible, enzymatic conversion of retinyl esters to retinol (for reviews see Harrison, 1998).

Retinyl ester-splitting enzymes are found ubiquitously, in many tissues, and it is generally assumed that their function, activity and regulation depend on the enzyme location. Whereas retinolliberating enzymes from pancreas and intestinal brush border are a pre-requisite for the full absorption of dietary vitamin A (Lombardo \& Guy, 1980; Rigtrup et al. 1994), isoenzymes in eyes provide free retinol as an intermediate in rhodopsin regeneration (Tsin \& Lam, 1986). In the liver, the central organ in maintaining body retinol homeostasis, the retinyl ester-converting enzymes are involved in the hydrolysis of vitamin A esters delivered to the hepatocytes via the receptor-mediated endocytosis of chylomicron remnants (Blomhoff et al. 1982) and the generation of free retinol from its storage form as esters (Goodman \& Blaner, 1984). The liberated retinol acts as precursor for re-esterification for storage (Blaner et al. 1985; Blomhoff et al. 1985), as a ligand for secretion with retinol-binding protein into the plasma (Smith et al. 1998), as an intermediate for the biosynthesis of retinoic acid (the final morphogenic derivative of vitamin A; Napoli \& Race, 1990) and as a substrate for oxidative reactions in the pathway for eliminating vitamin A from the body (Roberts et al. 1980). The steps in the synthesis of downstream metabolites of retinal (vitamin A aldehyde) are catalysed by cytochrome P450 proteins (Leo \& Lieber, 1985; Leo et al. 1987).

Vitamin A metabolism has been shown to be susceptible to therapeutic agents, and a few such interactions have been directly observed clinically, for example with the antibiotic procaine penicillin, which results in night blindness (Haubold et al. 1953). Recent studies carried out in vivo have shown that cefotiam and flupenthixol also can operate as vitamin A antagonists (Schindler et al. 2004). The broad-spectrum antibiotic cefotiam shares structural features with penicillins, and the thioxanthene drug flupenthixol has structural characteristics related to those of the phenothiazines. A comparison of the structures of both compounds reveals that cefotiam has a structural element in common with flupenthixol: a cationic residue. Another striking feature of these pharmacological agents is the fact that they are in vitro inhibitors of hepatic retinyl ester hydrolase (REH; Schindler, 2001).

We have, however, recently demonstrated that a number of therapeutic agents with REH inhibitory activity, including 
amiodarone (an antiarrhythmic agent) and trimeprazine (a phenothiazine neuroleptic drug), possess tertiary amine $\mathrm{N}$ atoms (Schindler, 2001). These findings raise the question of whether amiodarone and trimeprazine might also interfere with normal vitamin A metabolism in vivo. Against this background, amiodarone and trimeprazine were next selected to assess whether these compounds also adversely affect vitamin A status at doses comparable to those in clinical use.

Amiodarone is a prophylactic antiarrhythmic agent that prolongs the action potential duration in both atrial and ventricular myocardium (Dollery, 1991a). It was originally developed as an antianginal agent because of its vasodilatory actions. Nowadays, it is widely used for the management of various tachyarrhythmias and, to a lesser extent, of severe congestive heart failure. Unfortunately, its chronic use is complicated by a number of unwanted side-effects (Dollery, 1991a). The most common side-effects are reversible corneal opacities, changes in thyroid function, photodermatosis and pulmonary toxicity, but gastrointestinal complications, hepatic dysfunction, sleep disturbances and anorexia may occur. The usual recommended loading dosage for amiodarone is $200-600 \mathrm{mg} / \mathrm{d}$ (Mutschler \& Schäfer-Korting, 1996).

Amiodarone, an iodine-rich pharmacological agent, has the structure of 2-n-butyl-3[4-(2-diethylaminoethoxy)-3,5-diiodobenzoyl] benzofuran $\left(\mathrm{M}_{\mathrm{r}}\right.$ 645.3; Dollery, 1991a). It is a cationic amphiphilic drug (pKa 5.6; Dollery, 1991a) and possesses a large hydrophobic and a small hydrophilic tail that are believed to be involved in amiodarone-related toxicity. Another potentially important factor in the development of toxicity is the long elimination half-life of the antianginal drug, estimated at 26-107d (Physicians' Desk Reference, 1996).

Pharmacokinetic studies concerning the gastrointestinal absorption of amiodarone indicate that it is taken up by a simple diffusion process (Martin-Algarra et al. 1997). Intestinal uptake is slow, maximal levels being reached after $3-7 \mathrm{~h}$ (Dollery, 1991a). The peroral bioavailability of amiodarone is reported to range from about 20 to $80 \%$ (Martin-Algarra et al. 1997). In plasma, the protein binding of amiodarone at therapeutically achievable concentrations ranging from 1.5 to $4.0 \mu \mathrm{mol} / \mathrm{l}$ (Freedman \& Somberg, 1991) reaches $96 \%$ (Dollery, 1991a); this binding occurs predominantly with albumin but also with LDL and

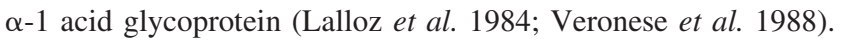

Amiodarone is metabolised intensively prior to its biliary elimination (Latini et al. 1984) and undergoes enterohepatic recirculation. $N$-deethylation to desethylamiodarone has been clearly established as the important metabolic pathway for amiodarone. In addition, the antiarrhythmic agent is metabolised by hydroxylation, $O$-dealkylation, deiodination and glucuronidation (Harris \& Roncucci, 1986).

Long-term treatment with amiodarone leads to an accumulation of the parent drug and its principal metabolite, desethylamiodarone. Both drugs are, however, preferentially distributed in, in decreasing order, the thyroid gland, lungs, kidneys, liver, heart, adipose tissue, skeletal muscle and brain (Morales et al. 2003). The ratio of desethylamiodarone to amiodarone in these tissues is less than 1, the exceptions being liver and lung tissue (Young \& Mehendale, 1986). Tissue amiodarone levels can generally be 100 times greater than those of blood (Freedman \& Somberg, 1991).

In addition, trimeprazine (known as alimemazine outside the UK, vallergan, nedeltran, panectyl, repeltin, temaril, theralene and variargil) was studied for its potency as a vitamin A antagonist, in as much as the structure of this compound has a number of features in common with that of amiodarone. Trimeprazine is an aliphatictype phenothiazine widely used clinically as an antipsychotic drug (Dollery, 1991b). It has pronounced sedative and antihistamine properties and some antimuscarinic actions (Dollery, 1991b). Trimeprazine may also be used as a cough suppressor (Kintz et al. 1995). The usual recommended daily dose is $5-40 \mathrm{mg}$ orally (Reynolds, 1989). The phenothiazinic drug is routinely given over courses of months to years and is well tolerated. Moreover, safety testing of trimeprazine in dogs has indicated that the drug has no end-organ toxicity (Dollery, 1991b). In rare cases, however, it can cause photosensitive dermatitis, drowsiness, abdominal discomfort and a reduction in the level of serum thyroid hormones, particularly thyroxine, in human subjects (Dollery, 1991b; Sauvage et al. 1999).

Like other members of the phenothiazine group, trimeprazine ( $N, N$-dimethyl-2-methyl-3-[phenothiazine-10-yl] propylamine) shares a related structural feature including two benzene rings bridged by $\mathrm{S}$ (position 5) and $\mathrm{N}$ (position 10) heteroatoms and a methyl-substituted three-C side chain on $N-10$ containing a tertiary amino group $\left(\mathrm{M}_{\mathrm{r}}\right.$ 298.44; Dollery, 1991b). Considering the chemical structure, it is to be expected that this neuroleptic agent might also behave like a cationic, amphiphilic drug. Unlike amiodarone, trimeprazine is a pharmacological agent for which there is little published pharmacokinetic information. When given orally, the absorption efficiency for the phenothiazine derivative is about $80 \%$ (Dollery, 1991b). Trimeprazine achieves peak plasma concentrations within $3 \cdot 5-4 \cdot 5 \mathrm{~h}$ after oral administration (Dollery, 1991b). The circulating drug is almost completely $(>90 \%)$ bound to plasma proteins (Dollery, 1991b).

Trimeprazine is associated with extremely low serum levels, probably owing to its concentration by various tissues in the body. The liver in particular may represent a sequestration compartment for phenothiazines (a liver:blood concentration ratio of up to 60:1; Coutselinis et al. 1974). Phenothiazines are predominantly eliminated in human subjects by hepatic biotransformation. The degradation products of trimeprazine metabolism are 3-hydroxytrimeprazine, trimeprazine sulphoxide and $\mathrm{N}$-desmethyltrimeprazine. These compounds are removed from the body by one of two mechanisms, excretion via the kidney being the first route of egress ( $75 \%$ ), followed by the gut (25\%; Kim \& Park, 1992).

The objective of the present experiments with Brown Norway rats was to investigate the individual effects of amiodarone and trimeprazine on the metabolism of vitamin A in vivo. In this paper, we report: (a) the postprandial plasma retinyl ester response; (b) the time course changes in the plasma retinol level throughout the $28 \mathrm{~d}$ exposure period; (c) the terminal observed hepatic REH activity; (d) the size and composition of the liver vitamin A stores; (e) the vitamin A content of the kidneys.

\section{Methods}

Sources of pharmacological agents, chemicals and experimental animals

Drugs were generously donated as follows: amiodarone hydrochloride by Sanofi Pharma (München, Germany) and trimeprazine by Heilit Arzneimittel (Reinbek, Germany). Retinyl palmitate was a kind gift from Hoffman-La Roche (Basel, Switzerland); stearoyl chloride, linoleoyl chloride, myristoyl chloride, and oleoyl chloride were from Sigma (Deisenhofen, Germany). The suppliers of other reagents and chemicals have been reported before 
(Schindler et al. 2002). All chemicals and solvents were of analytical grade.

Male Brown Norway rats (weight 214-356g) were obtained from the Zentralinstitut für Versuchstierzucht (Hannover, Germany). Upon arrival, rats were individually housed and kept in an environmentally controlled laboratory as described in our previous publication (Schindler et al. 2004). Tap water and nutritionally adequate diet (Rodent diet \#1314; Altromin, Lage, Germany), containing vitamin A (15000 IU vitamin A/kg diet), were available at all times except at the end (days 26 and 27) of the experiment. At the $27 \mathrm{~d}$ time point, rats were gavaged with retinyl palmitate, the predominant retinyl ester in food (Fig. 1). Body weights were determined daily throughout the testing period, and the drug dosage was adjusted if necessary. Guidelines for the ethical care and treatment of animals from the Animal Care and Use Committee at Christian-AlbrechtsUniversity Kiel were strictly followed.

\section{Assessments}

The observation and specimen collection schedule is shown in Fig. 1. The animals were observed daily for clinical signs of nutritional deficiency, toxicity or morbidity. For the 4-week study, eighteen rats were randomised into three groups that received long-term exposure by the oral route to amiodarone or trimeprazine or no drug as placebo group. Control animals were given an identical volume of benzyl alcohol (185 mM), the solvent for the drugs (see Table 1 for specific details). An oral treatment regimen was selected to mimic the clinical situation associated with therapy using the aforementioned drugs. The intragastric doses of the test compounds were arbitrarily selected as approximately representing the usual therapeutic range used clinically. To illustrate this, we compiled corresponding data for the study drugs from a review of the available literature (Table 1; Reynolds, 1989; Mutschler \& Schäfer-Korting, 1996). The drug doses employed were, however, calculated from the recommended daily therapeutic doses in the average $65 \mathrm{~kg}$ adult human subject corrected to relative body surface area according to Ungemach (Löscher et al. 1991). Each dose was administered via an intubation tube once daily for 4 weeks.

Experiments to determine whether the co-administration of esterified retinol and amiodarone or trimeprazine resulted in

\section{Time (d)}

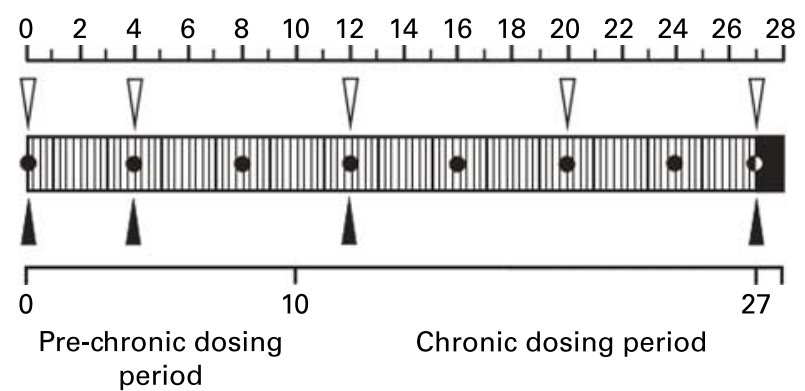

Fig. 1. Graphic presentation of the experimental design used in this study showing the progression of the dosing experiment as a function of time (pre-chronic dosing period days $0-8$, chronic dosing period days 12-27). [I], Drug treatment; $\nabla$, blood collection for the assessment of plasma clinical chemistry; - systematic retinol; $\boldsymbol{l}$, haematology; $\mathbf{C}$, vitamin A load test; blood drawn for measurement of the postprandial appearance of retinyl ester in plasma. incomplete oral vitamin A bioavailability were carried out as follows. Day 27 consisted of a $12 \mathrm{~h}$ period, starting with blood specimen collection in the morning after an overnight fast. At time zero, an intragastric dose of an amiodarone or trimeprazine solution was injected through the feeding tube, followed by $0.25 \mathrm{ml}$ virgin olive oil containing retinyl palmitate (total of $35 \mu \mathrm{g} / \mathrm{kg}$ body weight). The drug concentrations used were 40 and $1.0 \mathrm{mg} / \mathrm{kg}$ for amiodarone and trimeprazine, respectively (Table 1). An additional group of rats (controls) was given $1 \mathrm{ml}$ benzyl alcohol $(185 \mathrm{~mm})$ without a drug and received $0.25 \mathrm{ml}$ virgin olive oil containing retinyl palmitate (total of $35 \mu \mathrm{g} / \mathrm{kg}$ body weight) at identical time points. The tube was washed with virgin olive oil $(0.75 \mathrm{ml})$ to ensure delivery of all of the vitamin solution.

Blood samples for measuring the postprandial response in retinyl esters were collected into tubes containing sodium heparin 2, 4, 6, 8, 10 and $12 \mathrm{~h}$ after intragastric dosing via gavage (post-dose). The sum of retinyl esters was used for estimating vitamin A absorption. Plasma concentration-time data for retinyl esters were characterised using the following pharmacokinetic parameters: peak concentration, time to peak concentration and area under the retinyl ester plasma concentration $v$. time curve from zero to $12 \mathrm{~h}\left(\mathrm{AUC}_{0-12}\right)$. The values for peak concentration, time to peak concentration (a simple estimate of the speed of absorption) and $\mathrm{AUC}_{0-12}$ (determined by spline integration) were calculated from individual postprandial vitamin A ester curves.

At study termination (Fig. 1), the rats were killed by an overdose of anaesthetic, and blood, liver and kidneys were collected. The organs were rinsed with ice-cold saline containing $2.5 \mathrm{mmol}$ ascorbate/ 1 and $1.7 \mathrm{mmol}$ EDTA, blotted and weighed. All plasma and tissue samples were immediately frozen in liquid $\mathrm{N}_{2}$, before being stored in light-proof tubes at below $-20^{\circ} \mathrm{C}$ until analysis. Plasma and kidneys were analysed for free and esterified retinol. Liver was analysed for REH activity and vitamin A (retinol plus retinyl esters) content.

\section{Determination of retinyl ester hydrolase activity and protein concentration}

To measure hepatic REH activity, liver was extracted directly as follows: $2 \mathrm{~g}$ liver was homogenized on ice in a Potter-Elvehjem homogenizer (Braun; Melsungen, Germany) in five volumes of $0 \cdot 1 \mathrm{M}$-Tris-maleate buffer, $\mathrm{pH} 7 \cdot 0$. The emulsion was centrifuged at $50000 \mathrm{~g}$ for $5 \mathrm{~min}$ at $4^{\circ} \mathrm{C}$, and the clear supernatant was used to determine REH activity by measuring the rate of release of retinol from retinyl palmitate.

REH activity was determined according to a micro-method of Cooper and Olson (1986), as previously reported by our laboratory (Schindler et al. 1998). Reaction mixtures consisted of $0.5 \mathrm{ml}, 2 \mathrm{mg} / \mathrm{ml}$ Triton X-100, $200 \mathrm{~mm}-3$-[(3-cholamidopropyl)dimethylammonio]-1-propane-sulfonate, 0.05-mM retinyl palmitate and $0 \cdot 1 \mathrm{M}$-Tris-maleate, $\mathrm{pH}$ 7.2. 3-[(3-Cholamidopropyl)dimethylammonio]-1-propane-sulfonate was added to improve the solubility of the retinyl palmitate. The reaction was initiated with the enzyme sample $(2.4 \mathrm{mg}$ protein) and incubated for $60 \mathrm{~min}$ at $37^{\circ} \mathrm{C}$. After stopping the reaction with the addition of $0.5 \mathrm{ml}$ ice-cold ethanol, liberated retinol was extracted from the reaction mixtures with $2 \mathrm{ml} n$-hexane containing $0 \cdot 1 \%$ (w/v) butylated hydroxytoluene $(1 \mathrm{~g} / \mathrm{l})$. Extraction was performed in the dark at $25^{\circ} \mathrm{C}$. Following solvent removal, samples were resuspended in chloroform-methanol $(1 \cdot 5: 4, \mathrm{v} / \mathrm{v})$. The resulting mixture was 
Table 1. Oral drug administration protocols

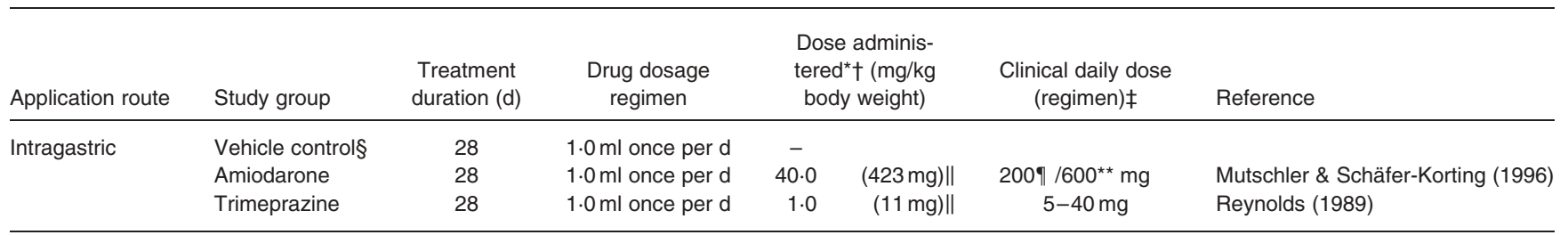

* The animals were weighed daily, and the dose was adjusted if necessary.

† Vehicle fluid for dose solution preparation was ( $185 \mathrm{~mm}$ ) benzyl alcohol.

‡Comparison of the therapeutically active doses as adapted from the literature (Reynolds, 1989; Mutschler \& Schäfer-Korting, 1996).

$\S$ Control rats received the dosing vehicle, i.e. benzyl alcohol ( $185 \mathrm{~mm}$ ) only.

|| Daily therapeutic dose in the average $65 \mathrm{~kg}$ adult human (in parentheses).

ๆ Maintenance dose.

** Loading dose every $12 \mathrm{~h}$ for 8-10d (Mutschler \& Schäfer-Korting, 1996).

then separated by HPLC equipped with a C-18 reverse-phase column, as described below for plasma retinol determination. In the above assay, the reaction rate was linear with respect to time and enzyme concentration. In each assay, blank tubes (without enzyme) were measured to correct for spontaneous hydrolysis of the substrate. Incubations were performed in a $\mathrm{N}$ atmosphere in the dark to prevent the photodecomposition of vitamin A. An average of duplicate or triplicate analyses of each sample was used for this enzymatic assay. The enzyme activity was defined as the amount of enzyme catalysing the release of $1 \mathrm{nmol}$ retinol/h per mg protein.

The protein concentration of the supernatant fraction was determined by the Bradford dye-binding method with bovine serum albumin as standard (Bradford, 1976).

\section{Extraction of tissue vitamin A (retinol plus retinyl esters)}

Vitamin A content was determined in post mortem biopsies from the liver and kidneys as detailed by Schindler et al. (1987). Liver tissue $(2 \mathrm{~g}$ ) or the whole kidneys in an equal volume of ice-cold physiologial saline $(150 \mathrm{mmol} \mathrm{NaCl} / \mathrm{l})$ containing ascorbate $(0.5 \mathrm{mg} / \mathrm{ml})$ and EDTA $(0.5 \mathrm{mg} / \mathrm{ml})$ were homogenised using an Ultra-Turrax homogeniser (Braun), and the homogenate was lyophilised. The dried tissue was extracted four times with $5 \mathrm{ml}$ chloroform-methanol $(1: 1, \mathrm{v} / \mathrm{v})$ containing butylated hydroxytoluene $(230 \mu \mathrm{mol} / \mathrm{l})$. Prior to being loaded on to an analytical HPLC column, an aliquot of the combined organic phase was diluted 3-fold with chloroform-methanol $(1: 3 \cdot 2, \mathrm{v} / \mathrm{v})$. In the case of the kidneys, vitamin A was extracted with five volumes of $n$-hexane containing butylated hydroxytoluene $(230 \mu \mathrm{mol} / \mathrm{l})$. The tissue extracts were then dried under $\mathrm{N}$, reconstituted in $500 \mu \mathrm{l}$ chloroform-methanol $(1 \cdot 7: 4, \mathrm{v} / \mathrm{v})$ and analysed directly by HPLC. The reverse-phase procedure was that used for determining plasma vitamin A. The amount of free and esterified retinol (i.e. total vitamin A) in the samples was expressed in terms of retinol equivalents per $\mathrm{g}$ tissue (wet weight).

\section{Estimation of plasma and tissue vitamin A levels}

Blood samples were taken by tail clip using sodium heparin as an anticoagulant, and plasma was separated via centrifugation at $1500 \mathrm{~g}$ for $20 \mathrm{~min}$ at $4^{\circ} \mathrm{C}$. Analyses of systemic vitamin A were performed on deproteinised, hexane-extracted samples as previously described (Schindler et al. 1985). After vacuum concentration and reconstitution of the residue in an appropriate solvent, the concentration of free and esterified retinol was determined fluorimetrically $\left(\lambda_{\mathrm{ex}}=325 \mathrm{~nm}, \lambda_{\mathrm{em}}=480 \mathrm{~nm}\right)$ by a validated reverse-phase HPLC procedure using a Bio-Tek Kontron spherisorb octadecylsilane $(5 \mathrm{~mm}$ internal diameter $\times 25 \mathrm{~cm})$ column (Bio-Tek Kontron, Neufahren, Germany) eluted with methanol at a flow-rate of $1.8 \mathrm{ml} / \mathrm{min}$ according to the procedures of Schindler et al. (1985). The standard curves were generated by injecting authentic retinoids in methanol by using a constant volume with increasing concentrations. Standards of vitamin A alcohol and retinol esterified to myristic, linoleic, palmitic, oleic and stearic acid were prepared in our laboratory according to known procedures (Ito et al. 1974; Huang \& Goodman, 1985). In pure standard solutions, retinol and retinyl esters were quantified using appropriate extinction coefficients $(1 \% / \mathrm{cm})$ from Schindler et al. (2002). Under our chromatographic conditions, however, myristate and linoleate esters comigrated, as did the palmitate and oleate esters. They were consequently calculated and represented as two fractions. The above procedure was that used for determining tissue vitamin A as well.

\section{Haematology and clinical chemistry}

Haematological characteristics (blood count, $\mathrm{Hb}$ and haematocrit) were examined in tail-tip blood. Plasma measurements of electrolytes $(\mathrm{Na}, \mathrm{K}, \mathrm{Cl})$, urea, creatinine and total plasma proteins were obtained by automated methodology with the Hitachi 911E System (Hitachi Clinical Diagnostic Systems, Berlin, Germany).

\section{Statistical analysis}

The experimental data were analysed as a completely randomised design with repeated measurement data for the traits systemic retinol and weight of rat, as indicated in Fig. 1. For modelling the covariance structure of the repeated measurement data, we used SAS procedure MIXED version 6.12, 1996 (SAS Institute Inc., Cary, NC, USA). A suitable structure type was heterogeneous compound symmetry, i.e. an equal correlation between repeated measurements within the same rat and an allowance for heterogeneous variances at each time of measurement. The analysed fixed effects were treatment (study group in Table 1) and time of measurement with corresponding effects of interaction (if repeated measurements were present). Because the weight of the rats differed between the treatment groups, a correction was made by analysis of covariance techniques with initial weight as the covariate. The results are reported here as 
estimated mean values with their standard errors; unless otherwise indicated, $P<0.05$ was regarded as significant.

\section{Results}

During the experiments, all the animals maintained good health and remained active, as determined by physical examination, periodic assessments of haematology (e.g. red blood cells, leucocytes, platelets, $\mathrm{Hb}$ and haematocrit) and routine laboratory examination of plasma electrolytes, blood urea, creatinine and total plasma proteins (data not shown). Regular weighings throughout this experiment revealed, however, that amiodarone treatment led to a decrease in body weight (Fig. 2). On completion of the study, the body weights of the rats that received amiodarone were significantly less than those of animals treated only with the vehicle $(P<0.009)$. Therefore, additional analyses were carried out to determine whether the weight loss induced by amiodarone was associated with gastrointestinal tract dysfunction.

To address this question, animals were evaluated for their ability to absorb vitamin A by measuring the post-absorptive response in plasma retinyl esters after oral bolus administration of the vitamin (as a tolerance test to detect fat-malabsorption states). The estimated mean plasma concentration $v$. time curves (tolerance curves) for the three groups are shown in Fig. 3, which shows that the plasma concentrations of retinyl ester after doses administered under conditions of amiodarone treatment were substantially lower than those after baseline conditions $(P<0 \cdot 05)$. None of the animals had measurable

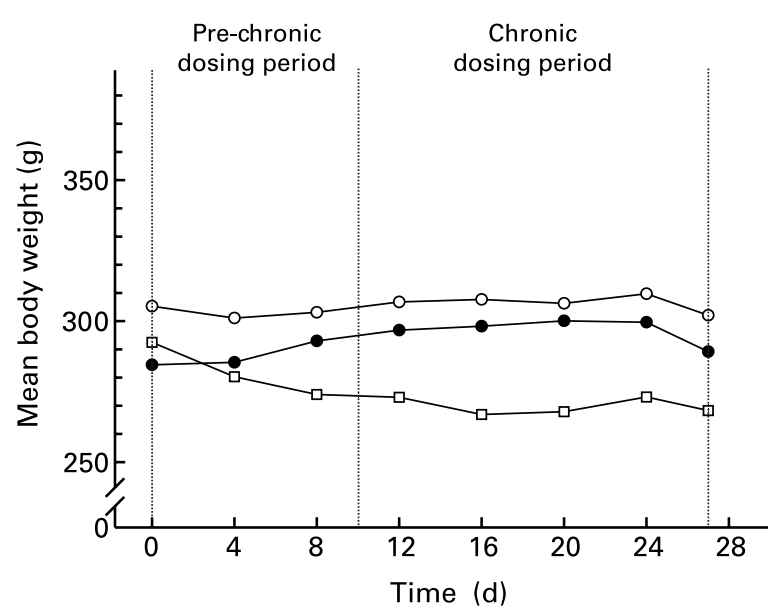

Fig. 2. Mean body weight changes for male Brown Norway rats throughout the 28-d study period. Eighteeen rats (initial body weight $214-356 \mathrm{~g}$ ) were allocated randomly to the following groups: dosing vehicle (benzyl alcohol, $185 \mathrm{~mm}$; ) amiodarone $(40 \mathrm{mg} / \mathrm{kg}$ body weight; $\square)$ or trimeprazine $(1.0 \mathrm{mg} / \mathrm{kg}$ body weight; $\bullet$ ). For clarity, means are shown for two time intervals (treatment as shown in Fig. 1); vertical dotted lines indicate the start of drug treatment (day 0 ), the beginning of chronic dosing (day 10), and the end of the exposure period (day 27). A significant main effect of time $(P<0.003)$ and a significant drug $\times$ time interaction $(P<0.001)$ were identified among all three treatments between days 0 and 8 . Furthermore, there was a significant drug $\times$ time interaction in both drug groups (for amiodarone, $P<0.001$; for trimeprazine, $P<0.003)$. Overall, the change in body weight from day $12-28$ also showed a significant main effect of time $(P<0.003)$. For trimeprazinetreated rats there was a significant drug $\times$ time interaction $(P<0.007)$ during the chronic dosing period. Amiodarone-dosed rat demonstrated a significant difference in body weight from both trimeprazine- $(P<0.0041)$ and placebotreated rats $(P<0.0041$ and $P<0.009$, respectively) from 12 day of exposure until the end of the study. ANOVA was used for repeated measures.

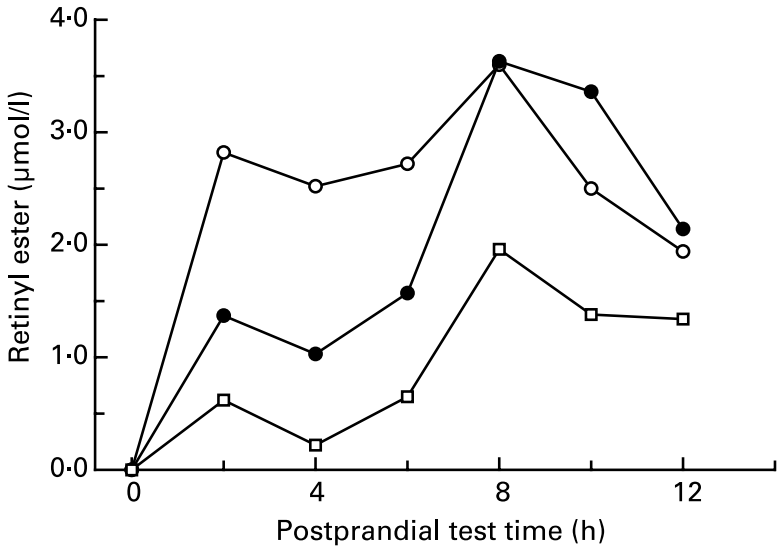

Fig. 3. Line plots of postprandial retinyl ester respone to an oral bolus administration of vitamin A in combination with dosing vehicle (benzyl alcohol, 185 estimated mean plasma retinyl ester concentration (i.e. observed values) for each group of six Brown Norway rats $v$. time. With the use of area under the curve from zero to $12 \mathrm{~h}$ as a respone variable in the statistical model, the effect of amiodarone-treatment was significantly different from that in animals that received either $185 \mathrm{~mm}$ benzyl alcohol $(P<0.041)$ or trimeprazine $(P=0.006)$.

concentrations of retinyl esters in their plasma at baseline. Rats in all groups showed a two-peak increase in plasma retinyl ester levels (one peak at $2 \mathrm{~h}$ and the other at $8 \mathrm{~h}$; Fig. 3, observed values). Specifically, the measured time to peak concentration was significantly greater $(P<0.02)$ in the amiodarone $(8.67($ SEM 0.66$)$ h) and trimeprazine $(9 \cdot 00$ (SEM $0 \cdot 66) \mathrm{h}$ ) groups compared with the group of control animals (6.33 (SEM 0.66) h). At the time when the second peak plasma concentration was obtained (time to peak concentration), the amiodarone (2.64 (SEM 0.71) $\mu \mathrm{mol}$ retinol equivalents/l), trimeprazine (4.63 (SEM 0.71) $\mu \mathrm{mol}$ retinol equivalents/l) and placebo (4.30 (SEM 0.71) $\mu \mathrm{mol}$ retinol equivalents/l) groups were not significantly different from each other in terms of the maximum mean plasma retinyl ester rise (peak concentration). However, the $\mathrm{AUC}_{0-12}$ of retinyl esters for the amiodarone-dosed animals at day $27(0.61$ $($ SEM $0 \cdot 22) \mu \mathrm{mol} \times \mathrm{h} / \mathrm{l})$ was significantly decreased $(P=0.006)$ compared with the $\mathrm{AUC}_{0-12}$ under control conditions (1.61 (SEM $0 \cdot 22) \mu \mathrm{mol} \times \mathrm{h} / \mathrm{l})$, but there was no significant change $(P=0.353)$ secondary to trimeprazine treatment $(1.31(\operatorname{SEM~} 0.22 \mu \mathrm{mol} \times \mathrm{h} / \mathrm{l})$.

Evidence for the action of the test drugs on vitamin $\mathrm{A}$ metabolism was also sought using serial measurements of systemic retinol level. Retinol balance was determined during a $10 \mathrm{~d}$ pre-chronic dosing period followed by a $17 \mathrm{~d}$ chronic dosing period (Fig. 4). The protocol defined acute treatment as one that took $10 \mathrm{~d}$ (interval $0-8 \mathrm{~d}$; Fig. 4), and chronic treatment as one that took more than $10 \mathrm{~d}$ (interval $12-28 \mathrm{~d}$; Fig. 4) to facilitate the comparison of our present results with previous ones (Schindler et al. 2004). Drug $\times$ time interaction terms were all significant for plasma retinol concentrations between days 0 and 8 after amiodarone $(P<0.001)$ and trimeprazine $(P<0.003)$, but the drug $\times$ time interaction term was not significant after dosing vehicle $(P=0 \cdot 254)$. The systemic retinol concentration was lower in the amiodarone $(P=0.4)$ and trimeprazine $(P=0.85)$ groups than the control group during the pre-chronic dosing period, but this difference was not significant.

During the chronic dosing period, there was a rise in plasma retinol concentration in the vehicle-treated and trimeprazine-treated rats, followed by a decline and by a further increase to reach 


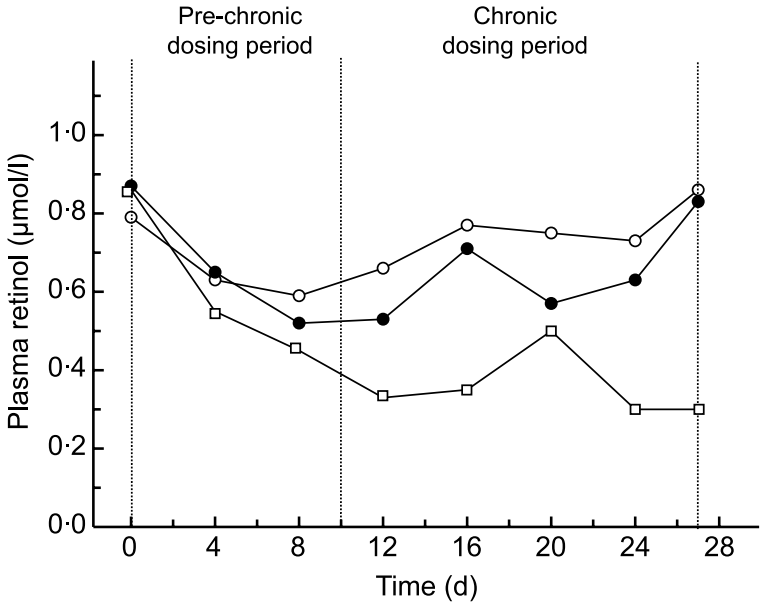

Fig. 4. Time course changes in the level of plasma retinol in Brown Norway rats. Separate groups $(n 6)$ of rats were administered by the enteral route $185 \mathrm{~mm}$ benzyl alcohol (dosing vehicle; 0 ), amiodarone $(40 \mathrm{mg} / \mathrm{kg}$ body weight; $\square$ ) or trimeprazine $(1.0 \mathrm{mg} / \mathrm{kg}$ body weight; $\bullet$ ) once daily for $28 \mathrm{~d}$. For clarity, estimated means are shown for two time intervals (blood collection and treatment as shown in Fig. 1); vertical dotted lines indicate the start of drug treatment (day 0), the beginning of chronic dosing (day 10), or the end of the exposure period (day 28). Overall, for systemic retinol measured on days 0,4 and 8 , a significant main effect of time $(P<0.001)$ and a significant drug $\times$ time interaction $(P<0.011)$ were observed. A significant main effect of drug $(P<0.001)$ was identified among all three treatments between days 12 and 28. For trimeprazine-treated rats there was a significant drug $\times$ time interaction $(P=0.009)$ during the chronic dosing period. Amiodarone-dosed rats demonstrated a significant difference in circulating retinol from both trimeprazine and placebo-treated rats $(P<0.001$ and $P<0.001$, respectively) from $12 \mathrm{~d}$ of exposure until the end of the study. ANOVA was used for repeated measures.

the baseline levels at the end of the experiments. In the treatment group being dosed with amiodarone, the pattern was similar but retinol concentrations peaked later $(20 \mathrm{~d} v .16 \mathrm{~d})$ and at a lower level than was seen with the other groups $(P<0 \cdot 0001)$. Except for trimeprazine $(P=0 \cdot 009)$, there was no further significant interaction between drug and time from days 12-28. Another important finding that emerged from this study was that amiodarone significantly prevented the increase in plasma retinol towards the pre-treatment values during the last part of the chronic dosing period.

Conceptually, a decrease in circulating retinol level could arise from inadequate vitamin A intake, impaired mobilisation, excessive losses or a combination of these factors. To ascertain more on the mechanism of action of the test compounds, we next examined the effects of the drugs on the various parameters of liver vitamin A. The results shown in Table 2 indicate that trimeprazine failed to significantly inhibit hepatic REH $(P<0 \cdot 13)$. However, the difference between the enzyme responses in the amiodarone and control groups approached statistical significance $(P=0 \cdot 06)$.

These in vivo results establish for the first time that REH activity is down regulated by amiodarone and trimeprazine. Table 2 also summarises the effects of drug administration on the size and composition (retinoid pattern) of liver vitamin A stores. There were significant decreases in the liver vitamin A content over time in each test group $(P<0 \cdot 02)$, the most substantial decrease being seen in the amiodarone group $(P<0.001)$. The relative amount of free retinol in the liver was increased by drug exposure when compared with vehicle-treated rats. However, the difference reached statistical significance only in the trimeprazine group $(P<0.025)$. A compositional analysis of retinyl esters revealed that amiodarone treatment increased $(P<0.001)$ proportions of retinyl linoleate and retinyl myrisate and decreased $(P<0.05)$ those of retinyl palmitate and retinyl oleate without any change in the retinyl stearate fraction.

As well as the liver, the concentrations of free and esterified retinol were also measured in the kidney (Table 2), which is a target organ for vitamin A. However, Brown Norway rats did not show abnormal levels of vitamin $\mathrm{A}$ in this extrahepatic tissue following oral dosing with amiodarone $(P=0.29)$ or trimeprazine $(P=0.7311)$. Thus, although plasma retinol levels were decreased in amiodarone-treated rats, retinol was transported efficiently to the peripheral tissues of these animals.

\section{Discussion}

This study demonstrates, for the first time, that clinically achievable doses of amiodarone and trimeprazine have the potential to affect adversely both the intestinal and the liver metabolism of

Table 2. The influence of oral dosing of amiodarone or trimeprazine on the specific activity of hepatic retinyl ester hydrolase, the size and composition of liver vitamin A stores and the retinoid content of the kidneys in Brown Norway rats ${ }^{*}+$ (Estimated mean value with their standard errors for six rats per group)

\begin{tabular}{|c|c|c|c|c|c|c|c|}
\hline \multirow[b]{2}{*}{ Tissue } & \multirow[b]{2}{*}{ Analyte } & \multicolumn{2}{|c|}{ Vehicle } & \multicolumn{2}{|c|}{ Amiodarone } & \multicolumn{2}{|c|}{ Trimeprazine } \\
\hline & & Mean & SEM & Mean & SEM & Mean & SEM \\
\hline \multirow[t]{6}{*}{ Liver } & $\mathrm{REH}^{*}$ & 4.5 & 0.4 & $3 \cdot 3$ & 0.38 & 3.6 & 0.4 \\
\hline & Vitamin A $(\mathrm{nmol} / \mathrm{g}) \S$ & $3422 \cdot 58^{a}$ & $189 \cdot 60$ & $2292 \cdot 8^{\mathrm{b}}$ & $179 \cdot 54$ & $2679 \cdot 34^{\mathrm{bc}}$ & 187.57 \\
\hline & Retinol (\%) & $13 \cdot 6^{\mathrm{a}}$ & 1.0 & $15 \cdot 4^{\mathrm{ab}}$ & 0.94 & $17 \cdot 2^{\mathrm{b}}$ & 0.98 \\
\hline & $\mathrm{RL} / \mathrm{RM}(\%)$ ๆ & $4 \cdot 9^{\mathrm{a}}$ & 0.73 & $10 \cdot 3^{b}$ & 0.68 & $5 \cdot 9^{a b}$ & 0.71 \\
\hline & $\mathrm{RP} / \mathrm{RO}(\%)$ & $64 \cdot 5^{a}$ & 1.77 & $59 \cdot 1$ & 1.67 & $61 \cdot 0^{\mathrm{ab}}$ & 1.93 \\
\hline & RS (\%) & $17 \cdot 1$ & 0.71 & $15 \cdot 3$ & 0.67 & $15 \cdot 8$ & 0.69 \\
\hline Kidney & Vitamin A $(\mathrm{nmol} / \mathrm{g}) \S$ & $4 \cdot 36$ & 0.52 & $2 \cdot 79$ & 0.49 & $4 \cdot 36$ & 0.49 \\
\hline
\end{tabular}

$\mathrm{REH}$, retinyl ester hydrolase; RL/RM, retinyl linoleate/retinyl myristate; RP/RO, retinyl palmitate/retinyl oleate; RS, retinyl stearate.

a,b,c Mean values in the same row with unlike superscript letters were significantly different $(P<0.05)$.

${ }^{*} \mathrm{REH}$ activity was determined by monitoring the rate of retinol formation (nmol/h per $\mathrm{mg}$ protein) at $37^{\circ} \mathrm{C}$ from $50 \mu$ mol retinyl palmitate/l in $0.5 \mathrm{ml}$ buffer (Tris-maleate $0.1 \mathrm{mmol} / \mathrm{l}) \quad$ Triton $\quad \mathrm{X}-100 \quad(2 \mathrm{mg} / \mathrm{ml})$, 3-((3-cholamidoprophyl)dimethylammonio)-1-propane-sulfonate $(200 \mathrm{mmol} / \mathrm{l}), \mathrm{pH} 7 \cdot 2)$

†For further details of diets and procedures, see pp. 677-678

$\S$ The amount of vitamin A (retinol + retinyl ester) in liver and kidney samples is expressed as nmol retinol equivalents/g fresh tissue.

If Results are expressed as the percentage of the total vitamin A fraction (taken as $100 \%$ ) of rat liver. 
vitamin A. The metabolic interaction of amiodarone with vitamin A was, however, more pronounced than that seen with trimeprazine. Because of this, and in view of the limited data concerning the pharmacological as well as toxicological properties of trimeprazine, the following discussion focuses on amiodarone as a vitamin A antagonist.

Results from the body weight measurements in the present study (Fig. 2) showed that only amiodarone led to a decrease in body weight. In principle, the cessation of growth could result simply from an amiodarone-induced attenuation of digestion and absorption (bioavailability) of dietary components, especially luminal lipids. To test this possibility, we used the postprandial appearance of retinyl esters in plasma triacylglycerol-rich lipoproteins as measures of the apparent intestinal absorption of lipid esters, including vitamin A. With regard to fat absorption, our results (Fig. 3) show that the retinyl ester response follows a biphasic curve, moderately elevated at $2 \mathrm{~h}$, dropping at $4 \mathrm{~h}$ and rising to greatest peak height at $8 \mathrm{~h}$. Such a pattern of change in plasma esterified retinol can be attributed to some stomach bypass because of the relatively large dosing volume used. The $\mathrm{AUC}_{0-12}$ value for retinyl esters with amiodarone was decreased significantly compared with the $\mathrm{AUC}_{0-12}$ value when vitamin $\mathrm{A}$ was given alone. The time to maximum plasma concentration for retinyl esters with amiodarone was also significantly increased compared with that for controls, suggesting delayed gastric emptying and prolonged absorption. Although trimeprazine delayed the time to peak retinyl ester concentration, it did not alter the $\mathrm{AUC}_{0-12}$ of esterified retinol.

The decrease in the $\mathrm{AUC}_{0-12}$ of esterified retinol secondary to the simultaneous administration of oral retinyl palmitate with amiodarone may indicate that this drug also interferes with the further intestinal metabolism of vitamin A. However, the precise mechanism underlying the amiodarone-mediated inhibition of retinol absorption remains unclear. The major dietary forms of preformed vitamin A are long-chain fatty acid esters of retinol (Schindler et al. 1987). The absorption of these lipid esters involves a series of interdependent steps that include release of vitamin A from the food matrix, solubilisation into mixed bile salt micelles, hydrolysis of esterified retinol, transfer into the intestinal mucosal cells, resynthesis of retinyl ester and incorporation into lymphatic lipoproteins, which facilitate transport into the liver for storage. Hypothetically, the multistep process of vitamin A absorption provides the opportunity for amiodarone to interfere with the retinyl ester cleavage reaction and/or translocation of retinol from microvillus to intracellular compartment.

The detergent-like nature of amiodarone is the basis of certain adverse effects of this agent and can explain the ability of this drug to disrupt normal membrane architecture as well as to inhibit various enzymes, including hepatic REH. It can be anticipated that this also applies to the pancreatic retinyl ester cleavage enzyme. Positive proof has nevertheless not been obtained. As described in more detail below, the inhibition of REH by amiodarone may be caused by rendering the substrate at the lipid-water interface less susceptible to the enzyme. Within this context, one has to recall that amiodarone is a cationic as well as an amphiphilic iodinated benzofuran derivative. These properties make the drug capable of entering into both lipophilic interactions with the substrate and electrostatic interactions with anionic interfaces, such as the polar headgroup regions of the substrate monolayer. Amiodarone could also inhibit enzymatic activity by a direct interaction with the polypeptide chain at the interface or by a modification of surface charge, as amiodarone is an ionised amine at neutral $\mathrm{pH}$.

Apart from the action of the secretory hydrolase(s), intraluminal vitamin A metabolism is governed by a brush border retinyl ester-converting enzyme (Rigtrup et al. 1994). A major mechanism for altering the activity of membrane-bound enzymes is modification of membrane fluidity. Amiodarone can, however, jeopardise membrane and/or tissue integrity and consequently decrease the activity of several membrane-associated enzymes of the gut wall (Sirajudeen et al. 2000). In addition to paths of action mentioned above, this mechanism might also participate in the adverse effect of amiodarone on vitamin A absorption.

The present findings, which revealed that the administration of amiodarone led to a decreased intestinal availability of vitamin A, suggest that the use of amiodarone may also be complicated by a low postprandial appearance of lipid-soluble vitamins per se. In addition, data revealed that the changes in body weight effected by amiodarone might be due to global energy restriction rather than a reduction in the intake of specific nutrients such as vitamin A, as evidenced by sufficient body stores of this vitamin at the end of the 4-week treatment period (see later). However, the possibility that changes in body weight seen with amiodarone may in part be attributed to a hypophagic effect of the drug cannot be ruled out.

In mammals, plasma vitamin A exists in two forms: esterified retinol associated mainly with chylomicra after meals (Blomhoff et al. 1982), and retinol bound to retinol-binding protein (Smith et al. 1998). Like intestinal uptake, the processes of chylomicron clearance, as well as the mobilisation of stored esterified retinol from intracellular lipid droplets, requires retinyl ester hydrolysis. With this in mind, amiodarone and trimeprazine were next tested for their ability to inhibit hepatic REH and retinol release from the liver (measured as plasma retinol levels over time). An explanation for the effects of amiodarone and trimeprazine on the tested parameters is offered.

As a reflection of gavage-related stress during the pre-chronic interval, significant decreases in plasma retinol concentration from baseline were observed, as anticipated (Schindler et al. 2004). The plasma retinol concentrations were, however, somewhat lower for those rats assigned to receive amiodarone or trimeprazine in acute administration than for control rats (Fig. 4). The greatest reduction in plasma retinol concentration occurred $28 \mathrm{~d}$ after chronic dosing with amiodarone, whereas in vehicle-treated controls and trimeprazine-exposed rats, plasma retinol had returned spontaneously toward normal values (the rise being considered to represent an adaptive response) within 4 weeks after starting treatment.

Because plasma retinol levels do not reflect changes in vitamin A intake until the liver stores of the vitamin become depleted (see Table 2 for the vitamin A status of the animals), the fluctuation in plasma retinol concentrations during the dosing period cannot be explained on the basis of changes in the absorptive capacity of the intestine. Plasma retinol is, however, almost completely bound to retinol-binding protein, and we assume that the initial $0 \cdot 35 \mu \mathrm{mol} / 1$ drop until day 12 resulted from a fall in the carrier protein caused by an impaired hepatic retinol-binding protein synthesis rate in the amiodarone-treated rats. Although this hypothesis is speculative and remains to be experimentally tested, studies documenting a link between decreased plasma retinol and nutritional factors, including protein malnutrition and/or a low-caloric intake, 
appear to support such a contention (Rechigl et al. 1962; Ingenbleek et al. 1975a,b; Gofferje, 1978; Shetty et al. 1979).

The fact that treatment with amiodarone for $20 \mathrm{~d}$ causes plasma retinol levels to fall again raises the possibility of an additional mechanism. It is not known what local tissue drug concentration is achieved in the vicinity of the hepatic REH in rats treated with amiodarone. However, this agent is highly concentrated in tissues (Freedman \& Somberg, 1991; Morales et al. 2003) so that, after multiple doses (day 20), amiodarone could achieve sufficient concentrations to inhibit REH within the hepatocytes and/or lipocytes (Ito cells, hepatic stellate cells). It is therefore probable that the availability of the vitamin from its hepatic stores is affected by long-term treatment with amiodarone. This hypothesis can be supported by the finding that decreased plasma retinol levels (day 28) were accompanied by decreased REH activity in the liver of amiodarone-exposed rats (Table 2).

On the other hand, it can be seen that there was a tendency for the retinyl ester-cleaving activity to increase at the end of this period in the trimeprazine group. One mechanism underlying the rise in REH activity might be an induction of enzyme protein in these animals. However, the increased biosynthesis of this hydrolase probably represents a compensatory mechanism resulting from its inhibition. These findings led us to speculate that the control of retinol release from the liver might directly involve REH. The postprandial plasma retinyl palmitate response of the drug-treated groups was consistently and significantly less than the response of the control group, giving no indication that hepatic REH also plays a role in processes of chylomicron clearance.

As a reflection of retinyl ester malassimilation during the treatment interval, significant decreases in the hepatic vitamin A concentration induced by the test drugs were observed in the present study. Moreover, the hepatic retinol content of the amiodaroneexposed $(2.93 \mu \mathrm{mol} /$ total liver $)$ and trimeprazine-treated (3.91 $\mu \mathrm{mol} /$ total liver) rats was lower than that of the rats that did not receive drugs $(3.96 \mu \mathrm{mol} /$ total liver $)$, when the data were expressed on an absolute basis but not on a relative basis (see Table 2). The tendency towards lower hepatic retinol levels in the drug groups than in the control group again validates the hypothesis that the mobilisation of vitamin A from liver storage sites is facilitated by REH. Changes in hepatic retinyl ester composition require a concerted adaptation of vitamin A metabolism, adjusted by hydrolytic and synthetic activities for esterified retinol. Therefore, this and previous studies (Schindler et al. 2002, 2004) also sought to determine whether in vivo dosing with REH inhibitors could produce subsequent alterations in the liver vitamin A ester profile. The major finding of this investigation was that treatment of Brown Norway rats with amiodarone caused a considerable increase in the relative amounts of retinyl linoleate and retinyl myristate, mainly at the expense of the retinyl palmitate and retinyl oleate fraction, whereas trimeprazine failed to show such an effect.

Although our data suggest that REH was responsible for the observed pattern of retinol and its esters, they do not provide final proof for this concept. Mammalian liver contains several forms of retinol-liberating enzyme, each with its own particular properties, substrate and inhibitor specificities, and cellular distributions. The question of the role of REH raised by the above observations may be partly answered when the results of ongoing studies to determine substrate specificity profiles of individual retinyl ester cleavage enzymes are known.

There are clinical implications of the present findings that should be noted. The eye is known as a tissue in which amio- darone, trimeprazine and vitamin A accumulate. Because of this, and given that REH is sensitive to drug treatment, we must seriously consider the possibility that the cellular level of retinol in ocular tissue is under the control of these compounds. Vitamin A is, however, an eye-protecting agent (Congdon \& West, 1999; Yeum et al. 1999). Therefore, patients receiving chronic therapy with amiodarone or trimeprazine may suffer from cataracts (Edler et al. 1971; Flach et al. 1983) as a consequence of disturbances in the equilibrium between retinol and retinyl ester.

What is new in our findings is that amiodarone is a more potent vitamin A antagonist than trimeprazine. Nevertheless, both drugs are so widely used in disease therapy that a knowledge of their vitamin A incompatibility could be of benefit to all clinicians. In clinical praxis, multiple concomitant medication is usually given when high-potency pharmacological agents such as amiodarone and trimeprazine are administered. Amiodarone and trimeprazine, like some of the co-administered drugs, are also used chronically, and a potential for both xenobiotic-vitamin A and drug-drug interaction exists. In some instances, such interactions may lead to vitamin A deficiency secondary to a loss of vitamin A during digestion and absorption.

\section{References}

Blaner WS, Hendriks HFJ, Brouwer A, Leeuw AM, Knook DL \& Goodman DS (1985) Retinoids, retinoid-binding proteins, and retinyl palmitate hydrolase distribution in different types of rat liver cells. $J$ Lipid Res 26, 1241-1251.

Blomhoff R, Helgerud P, Rasmussen M, Berg T \& Norum KR (1982) In vivo uptake of chylomicron $\left({ }^{3} \mathrm{H}\right)$ retinyl ester by rat liver: evidence for retinol transfer from parenchymal to nonparenchymal cells. Proc Natl Acad Sci 79, 7326-7330.

Blomhoff R, Rasmussen M, Nilsson A, et al. (1985) Hepatic retinol metabolism. J Biol Chem 260, 13560-13565.

Bradford MM (1976) Rapid and sensitive method for quantitation of microgram quantities of protein utilizing the principle of protein-dye binding. Anal Biochem 72, 248-254.

Congdon NG \& West KP (1999) Nutrition and the eye. Curr Opinion Ophthalmol 10, 464-473.

Cooper DA \& Olson JA (1986) Properties of liver retinyl ester hydrolase in young pigs. Biochim Biophys Acta 884, 251-258.

Coutselinis A, Dimopoulos G \& Dritsas C (1974) Fatal intoxication with chlorpromazine with special regard to the influence of putrefaction on its toxicological analysis. Forensic Sci 4, 191-194.

Dollery C (1991a) Therapeutic Drugs, vol. 1, pp. A90-A94. Edinburgh: Churchill Livingstone.

Dollery C (1991b) Therapeutic Drugs, vol. 2, pp. T141-T145. Edinburgh: Churchill Livingstone.

Edler K, Gottfries G, Haslund J \& Ravn J (1971) Eye changes in connection with neuroleptic treatment especially concerning phenothiazines and thioxanthenes. Acta Psychiatr Scand 47, 377-384.

Flach AJ, Dolan BJ, Sudduth B \& Weddell J (1983) Amiodarone-induced lens opacities. Arch Ophthalmol 101, 1554-1556.

Freedman MD \& Somberg JC (1991) Pharmacology and pharmacokinetics of amiodarone. J Clin Pharmacol 31, 1061-1069.

Gofferje H (1978) Prealbumin and retinol-binding protein - highly sensitive parameters for the nutritional state in respect of protein. Med Lab 5, 38-44.

Goodman DS \& Blaner WS (1984) Biosynthesis, absorption, and hepatic metabolism of retinol. In The Retinoids, vol. 2, pp. 1-39 [AB Sporn, MB Roberts and DS Goodman, editors]. Orlando, FL: Academic Press.

Harris L \& Roncucci R (1986) Amiodarone. Paris: Médecine et Science Internationales. 
Harrison EH (1998) Lipases and carboxylesterases: possible roles in the hepatic metabolism of retinol. Аnnu Rev Nutr 18, 259-276.

Haubold H, Loew W \& Kolb E (1953) Einwirkung von Penicillin auf den Vitamin A -Gehalt des Serums sowie auf die Nachtsichtigkeit. Münchner Med Wochenschr 95, 792-796.

Helgerud P, Petersen LB \& Norum KR (1982) Acyl CoA: retinol acyltransferase in rat small intestine: its activity and some properties of the enzymic reaction. J Lipid Res 23, 609-618.

Huang HS \& Goodman DS (1985) Vitamin A and carotenoids. J Biol Chem 240, 2839-2844.

Ingenbleek Y, van den Schrieck HG, de Nayer P \& de Visscher M (1975a) The role of retinol-binding protein in protein-caloric malnutrition. Metabolis 24, 633-641.

Ingenbleek Y, van den Schrieck HG, de Nayer P \& de Visscher M (1975b) Albumin, transferrin and the thyroxine-binding prealbumin/retinolbinding protein complex in assessment of malnutrition. Clin Chim Acta 63, 61-67.

Ito YL, Zile M, Ahrens H \& DeLuca HF (1974) Liquid-gel partition chromatography of vitamin A compounds: formation of retinoic acid from retinyl acetate in vivo. J Lipid Res 15, 517-524.

Kim T-J \& Park J-S (1992) Identification of new urinary metabolites of trimeprazine in rats by gas chromatography-mass spectrometry. J Chromatogr 575, 295-300.

Kintz P, Berthault F, Tracqui A \& Mangin P (1995) A fatal case of alimemazine poisoning. J Anal Toxicol 19, 591-593.

Lalloz M, Byfield P \& Himsworth R (1984) Binding of amiodarone by serum proteins and the effects of drugs, hormones, and other interacting ligands. J Pharm Pharmacol 36, 366-372.

Latini R, Tognoni G \& Kates C (1984) Clinical pharmacokinetics of amiodarone. Clin Pharmacokinet 9, 136-156.

Leo MA \& Lieber CS (1985) New pathways for retinol metabolism in liver microsomes. J Biol Chem 260, 5228-5231.

Leo MA, Lowe N \& Lieber CS (1987) Potentiation of ethanol-induced hepatic vitamin A depletion by phenobarbital and butylated hydroxytoluene. J Nutr 117, 70-76.

Lombardo D \& Guy O (1980) Studies on the substrate specificity of a carboxyl ester hydrolase from human pancreatic juice. II. Action on cholesterol esters and lipid-soluble vitamin esters. Biochim Biophys Acta 611, 147-155.

Löscher W, Ungemach FR \& Kroker R (1991) Grundlagen der Pharmakotherapie bei Haus- und Nutztieren. Berlin: Verlag Paul Parey.

MacDonald PN \& Ong DE (1988) Evidence for a lecithin-retinol acyltransferase activity in the rat small intestine. J Biol Chem 263, $12478-12482$.

Martin-Algarra RV, Pascual-Costa RM, Merino M \& Casabo VG (1997) Intestinal absorption kinetics of amiodarone in rat small intestine. Biopharm Drug Dis 18, 523-532.

Morales AI, Barata JD, Bruges M, Arevalo MA, Gonzalez de Buitrage JM, Palma P, Branco P \& Perez-Barriocanal F (2003) Acute renal toxic effects of amiodarone in rats. Pharmacol Toxicol 92, 39-42.

Mutschler E \& Schäfer-Korting M (1996) Arzneimittelwirkungen, 7th ed., Stuttgart: Wissenschaftliche Verlagsgesellschaft

Napoli JL \& Race KR (1990) Microsomes convert retinol and retinal into retinoic acid and interfere in the conversion catalyzed by cytosol Biochim Biophys Acta 1034, 228-232.
Physicians' Desk Reference (1996) Montvale: Medical Economics.

Rechigl M, Berger S, Loosli JK \& Williams HH (1962) Dietary protein and utilization of vitamin A. J Nutr 76, 435-440.

Reynolds JEF (1989) Martindale - The Extra Pharmacopeia. London: Pharmaceutical Press.

Rigtrup KM, McEwen LR, Said HM \& Ong DE (1994) Retinyl ester hydrolytic activity associated with human intestinal brush border membranes. Am J Clin Nutr 60, 111-116.

Roberts AB, Lamb LC \& Sporn MB (1980) Metabolism of all-trans retinoic acid in hamster liver microsomes. Oxidation of 4-hydroxy to 4-keto retinoic acid. Arch Biochem Biophys 199, 374-383.

Sauvage MF, Rousseau A, Marquet P, Dumeirain F, Raby C \& Lachâtre G (1999) In vitro and in vivo study of the antithyroid side effects of trimeprazine. Toxicol Appl Pharmacol 158, 125-131.

Schindler R (2001) Inhibition of purified pig and human liver retinyl ester hydrolase by pharmacologic agents. Lipids 36, 543-548.

Schindler R, Fielenbach T \& Rave G (2004) Flupenthixol and cefotiam: effects on vitamin A metabolism in rats. Brit $J$ Nutr 92 , 597-605.

Schindler R, Fielenbach T, Rave G, Blömer A \& Kellersmann R (2002) Vitamin A metabolism is altered in Brown Norway and Long-Evans rats infused with naftidrofuryl or erythromycin intravenously. Int $J$ Vitam Nutr Res 72, 210-220.

Schindler R, Klopp A, Gorny C \& Feldheim W (1985) Comparison between three fluorometric micromethods for determination of vitamin A in serum. Internat $J$ Vitam Nutr Res 55, 25-34.

Schindler R, Mentlein R \& Feldheim W (1998) Purification and characterization of retinyl ester hydrolase as a member of the nonspecific carboxylesterase supergene family. Eur $J$ Biochem 251, $863-873$.

Schindler R, Scholz M \& Feldheim W (1987) Determination of vitamin A in liver sausage and liver tissue from slaughtered animals using HPLC. Z Lebensm Unters Forsch 185, 208-212.

Shetty PS, Watrasiewics KE, Jung RT \& James WPT (1979) Rapid-turnover transport protein: an index of subclinical protein-energy malnutrition. Lancet ii, 230-232.

Sirajudeen KNS, Selvi RT, Babakrishnan DH \& Devaraj NS (2000) Effect of amiodarone on the membrane bound enzymes of rat intestine. Drug Chem Toxicol 23, 387-400.

Smith JE, DeMoor LM, Green EL \& Ritter SJ (1998) The complex between retinol and retinol-binding protein is formed in the rough microsomes of liver following repletion of vitamin A-depleted rats. Biochem Biophys Acta 1380, 10-20.

Tsin ATC \& Lam K-W (1986) Retinyl palmitate hydrolase activity in the bovine retina. Biochem Biophys Res Comm 134, 1209-1214.

Veronese ME, McLean S \& Hendricks R (1988) Plasma protein binding of amiodarone in a patient population: measurement by erythrocyte partitioning and a novel glass-binding method. Br J Clin Pharmacol 26, $721-731$.

Yeum K-J, Shang F, Schalch W, Russell RM \& Taylor A (1999) Fat-soluble nutrient concentrations in different layers of human cataractous lens. Curr Eye Res 19, 502-505.

Young RA \& Mehendale HM (1986) In vitro metabolism of amiodarone by rabbit and rat liver and small intestine. Drug Metabol Dispos 14, $423-429$. 